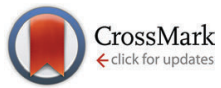

Cite this: J. Mater. Chem. C, 2016, 4, 1871

Received 11th December 2015, Accepted 28th January 2016

DOI: $10.1039 / \mathrm{c} 5 \mathrm{tc0} 4217 \mathrm{~h}$

www.rsc.org/MaterialsC

\section{The impact of charge transfer and structural disorder on the thermoelectric properties of cobalt intercalated $\mathrm{TiS}_{2} \dagger$}

\author{
Gabin Guélou, ${ }^{a}$ Paz Vaqueiro, ${ }^{a}$ Jesús Prado-Gonjal, ${ }^{a}$ Tristan Barbier, ${ }^{b}$ \\ Sylvie Hébert, ${ }^{\mathrm{b}}$ Emmanuel Guilmeau, ${ }^{\mathrm{b}}$ Winfried Kockelmann ${ }^{\mathrm{c}}$ and \\ Anthony V. Powell*a
}

\begin{abstract}
A family of phases, $\mathrm{Co}_{x} \mathrm{TiS}_{2}(0 \leq x \leq 0.75)$ has been prepared and characterised by powder $\mathrm{X}$-ray and neutron diffraction, electrical and thermal transport property measurements, thermal analysis and SQUID magnetometry. With increasing cobalt content, the structure evolves from a disordered arrangement of cobalt ions in octahedral sites located in the van der Waals' gap $(x \leq 0.2)$, through three different ordered vacancy phases, to a second disordered phase at $x \geq 0.67$. Powder neutron diffraction reveals that both octahedral and tetrahedral inter-layer sites are occupied in $\mathrm{CO}_{0.67} \mathrm{TiS}_{2}$. Charge transfer from the cobalt guest to the $\mathrm{TiS}_{2}$ host affords a systematic tuning of the electrical and thermal transport properties. At low levels of cobalt intercalation $(x<0.1)$, the charge transfer increases the electrical conductivity sufficiently to offset the concomitant reduction in $|S|$. This, together with a reduction in the overall thermal conductivity leads to thermoelectric figures of merit that are $25 \%$ higher than that of $\mathrm{TiS}_{2}, Z T$ reaching 0.30 at $573 \mathrm{~K}$ for $\mathrm{Co}_{x} \mathrm{TiS}_{2}$ with $0.04 \leq x \leq 0.08$. Whilst the electrical conductivity is further increased at higher cobalt contents, the reduction in $|S|$ is more marked due to the higher charge carrier concentration. Furthermore both the charge carrier and lattice contributions to the thermal conductivity are increased in the electrically conductive ordered-vacancy phases, with the result that the thermoelectric performance is significantly degraded. These results illustrate the competition between the effects of charge transfer from guest to host and the disorder generated when cobalt cations are incorporated in the inter-layer space.
\end{abstract}

\section{Introduction}

$\mathrm{TiS}_{2}$ is one of a number of transition-metal dichalcogenides whose structures consist of two-dimensional metal-chalcogen blocks, separated by a van der Waals' gap. $\mathrm{TiS}_{2}$ adopts the $1 \mathrm{~T}$ structure (space group $P \overline{3} m 1$ ) consisting of two-dimensional slabs of edge-linked TiS $_{6}$ octahedra (Fig. 1). The van der Waals' gap between successive $\mathrm{TiS}_{2}$ blocks contains a network of vacant octahedral and tetrahedral sites. The layered structure of $\mathrm{TiS}_{2}$ permits the ready intercalation of a wide range of guest species, including monatomic cations, ${ }^{1}$ molecular ions ${ }^{2,3}$ and organic molecules. ${ }^{4}$ Incomplete occupation of the network of

\footnotetext{
${ }^{a}$ Department of Chemistry, University of Reading, Whiteknights, Reading RG6 6AD, UK. E-mail: a.v.powell@reading.ac.uk

${ }^{b}$ Laboratoire CRISMAT, UMR6508 CNRS ENSICAEN, 6 bd Marechal Juin, 14050 Caen Cedex 4, France

${ }^{c}$ STFC, Rutherford Appleton Laboratory, ISIS Facility, Didcot OX11 OQX, UK $\dagger$ Electronic supplementary information (ESI) available: Powder X-ray diffraction data, thermal analysis data and additional magnetic and transport property data. See DOI: $10.1039 / \mathrm{c} 5 \mathrm{tc} 04217 \mathrm{~h}$
}
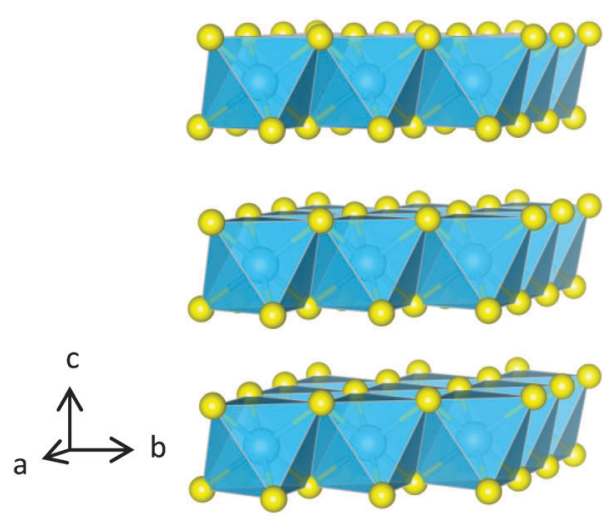

Fig. 1 The structure of $1 \mathrm{~T}-\mathrm{TiS}_{2}$ comprised of Ti-centered octahedra, shown in blue, with sulphur anions in yellow.

octahedral sites in the van der Waals' gap of dichalcogenides by monatomic guest species may give rise to a variety of twodimensional superstructures (Fig. 2) in phases of general formula $\mathrm{A}_{x} \mathrm{MS}_{2}(x<1.0)$. Furthermore, the incorporation of a guest species, 

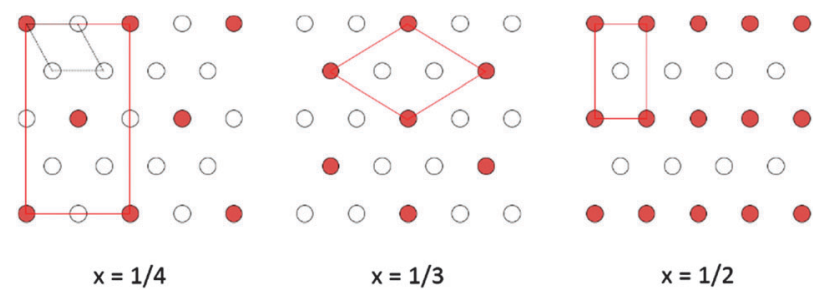

Fig. 2 Possible 2-dimensional superstructures of $A_{x} M_{2}$ for incomplete $(x<1)$ occupation of octahedral sites in the van der Waals' gap. The dashed line shows the primitive hexagonal cell of the pristine $(x=0)$ phase, whilst the red line outlines the supercells.

A, is accompanied by the transfer of electronic charge from guest to host. This provides a means of tuning the electron-transport properties. The presence of guest ions may also induce changes in the lattice component of the thermal conductivity. In addition to the important insights into the complex interplay of composition, structure and properties that such phases provide, the capacity to control the physical properties of a layered disulphide through intercalation offers considerable attractions in the design of new thermoelectric materials for energy harvesting.

As an all solid-state technology, thermoelectric energy harvesting offers advantages in terms of reliability, providing issues of cost and efficiency can be resolved. The efficiency of a device is determined by the transport properties of the materials of which it is comprised, embodied in a figure of merit $Z T=S^{2} \sigma T / \kappa$, where $S, \sigma$ and $\kappa$ are the Seebeck coefficient, electrical and thermal conductivities respectively. ${ }^{5}$ Doped-derivatives of $\mathrm{Bi}_{2} \mathrm{Te}_{3}$ that form the basis of commercial devices, exhibit a figure of merit, $Z T$, that approaches unity at $c a .380 \mathrm{~K} .{ }^{6}$ However, in addition to issues over the toxicity of tellurium, the relative scarcity of this element limits the opportunities for wide-scale implementation of thermoelectric technology. This has motivated the search for materials with technologically-viable thermoelectric performance that contain earth-abundant elements. It has been reported ${ }^{7}$ that the value of the thermoelectric power factor of $\mathrm{TiS}_{2}$ single crystals $\left(S^{2} \sigma=\right.$ $3.71 \mathrm{~mW} \mathrm{~m}^{-1} \mathrm{~K}^{-2}$ at $300 \mathrm{~K}$ within the $a b$ plane) is comparable with that of $\mathrm{Bi}_{2} \mathrm{Te}_{3}$, whereas the thermal conductivity is significantly higher, thereby reducing the figure of merit. The beneficial effects for thermoelectric performance of the low dimensionality of $\mathrm{TiS}_{2}$ coupled with the capacity to tune transport properties by intercalation has led to considerable interest in the layered disulphide, $\mathrm{TiS}_{2}$, as a candidate thermoelectric material. ${ }^{4,8-11}$

In this work, we have investigated the effects of cobalt incorporation on the electronic, magnetic and thermal properties of $\mathrm{TiS}_{2}$. The results demonstrate that there is a balance to be struck between the benefits of electron transfer in increasing the electrical conductivity and the deleterious impact on the Seebeck coefficient of the increase in charge-carrier density on intercalation. We show that the most promising thermoelectric properties are realised at relatively low levels of cobalt incorporation, where cobalt cations are disordered over the available interlayer octahedral sites. The figure of merit at these levels of cobalt incorporation is $c a .25 \%$ higher than that of the binary $\mathrm{TiS}_{2}$, reaching values of $Z T=0.30$ at $573 \mathrm{~K}$. Such materials are therefore of interest for energy recovery from low-grade waste heat at temperatures up to $600 \mathrm{~K}$, for which, despite the spectacular increases in $Z T$ achieved at high temperatures, there remains a dearth of suitable low-cost materials.

\section{Experimental}

$\mathrm{TiS}_{2}$ was synthesised from the elements Ti (Alfa Aesar, 325 mesh, 99.99\%) and S (Sigma Aldrich, flakes, 99.99\%). The sulphur flakes were dried at room temperature under vacuum before use. Stoichiometric amounts of the elements were ground together before being placed in a fused silica ampoule. This was evacuated to $10^{-4}$ Torr before sealing. The sealed ampoule was placed in a furnace and heated at $350^{\circ} \mathrm{C}$ for $4 \mathrm{~h}$ and $650^{\circ} \mathrm{C}$ for $12 \mathrm{~h}$, using a heating and cooling rate of $1{ }^{\circ} \mathrm{C} \mathrm{min}{ }^{-1}$. Samples of $\mathrm{Co}_{x} \mathrm{TiS}_{2}$ $(x=0.02 ; 0.04 ; 0.06 ; 0.08 ; 0.1 ; 0.15 ; 0.2 ; 1 / 4 ; 0.3 ; 1 / 3 ; 0.4 ; 1 / 2 ; 2 / 3$; 3/4) were prepared by grinding appropriate quantities of $\mathrm{TiS}_{2}$ and Co (Alfa Aesar, 99.99\%) prior to heating in an evacuated, sealed fused-silica ampoule. The mixture was heated at $650{ }^{\circ} \mathrm{C}$ for 2 successive periods of $48 \mathrm{~h}$ (heating/cooling rate $1{ }^{\circ} \mathrm{C} \mathrm{min}^{-1}$ ) with an intermediate regrinding. For both the $\mathrm{TiS}_{2}$ synthesis and the cobalt intercalation step it is critically important to use a slow cooling rate to allow the sulphur to reintegrate into the powder without condensing on the wall of the tube.

Powder X-ray diffraction data for all samples were collected using a Bruker D8 Advance Powder X-ray diffractometer, operating with Ge monochromated $\mathrm{CuK} \alpha_{1}$ radiation $(\lambda=1.54046 \AA)$ and fitted with a LynxEye detector. Data were collected over the angular range $5 \leq 2 \theta / /^{\circ} \leq 120$ for a period of 6 hours. Time-offlight powder neutron diffraction data for selected samples of $\mathrm{Co}_{x} \mathrm{TiS}_{2}(x=0.2,1 / 4,1 / 3,1 / 2$ and $2 / 3)$ were collected using the GEM diffractometer at the ISIS Facility, Rutherford Appleton Laboratory, UK. Data were collected for samples contained in thinwalled vanadium cans at room temperature and at temperatures to $2 \mathrm{~K}$, using a vanadium-tailed cryostat. Initial data manipulation and reduction, including the subtraction of the empty cryostat signal for low-temperature measurements was carried out using the Mantid software package ${ }^{12}$ Rietveld analysis of both powder X-ray and neutron diffraction data was carried out using the GSAS package ${ }^{13}$ and residual maps plotted with DRAWxtl. ${ }^{14}$

Magnetic susceptibility data for $\mathrm{Co}_{x} \mathrm{TiS}_{2}(x=0.2,1 / 4,1 / 3,1 / 2$ and 2/3) were obtained using a Quantum Design MPMS XL magnetometer. Data were collected over the temperature range $2.5 \leq T / \mathrm{K} \leq 298$ both after zero-field cooling (ZFC) and after cooling in the measuring field of $0.01 \mathrm{~T}$ (FC). Samples of $\mathrm{Co}_{x} \mathrm{TiS}_{2}$ for transport property measurements were consolidated using a hot press constructed in house. Powders were loaded into a graphite mould and pressed using tungsten carbide dies at $100 \mathrm{MPa}$ for $30 \mathrm{~min}$ at $903 \mathrm{~K}$ under a $\mathrm{N}_{2}$ atmosphere. Prior to consolidation, ball-milling was used to homogenise and reduce the particle size of the powders. Ball milling was carried out in a $25 \mathrm{~mL}$ stainless-steel jar with $6 \mathrm{~mm}$ stainless-steel balls, under an argon atmosphere in a Retsch Planetary Ball Mill PM100 at 350 rpm for 1 hour with 5 min interval steps, with a change of direction. The weight ratio of powder to balls was $3: 10$. Consolidation produced high density compacts with $\geq 95 \%$ of the crystallographic value for 


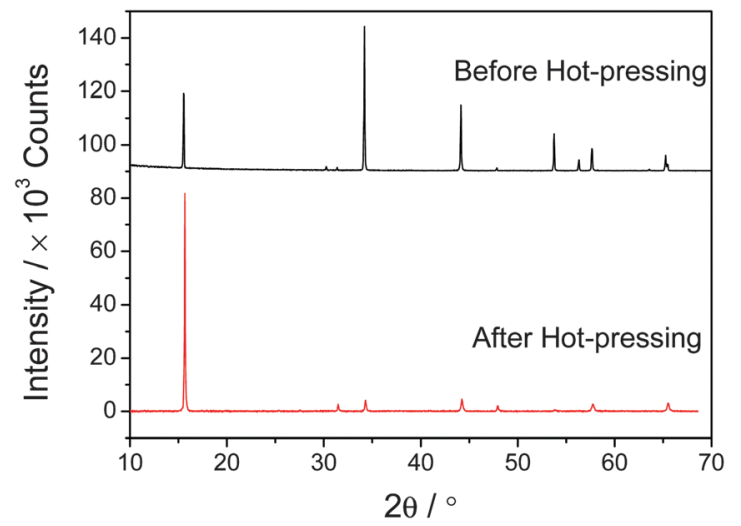

Fig. $3 \mathrm{X}$-ray diffraction patterns of $\mathrm{TiS}_{2}$ powder prior to hot pressing and the $\mathrm{TiS}_{2}$ pellet following hot-pressing, illustrating the preferred orientation in the consolidated sample.

low cobalt contents $(x \leq 0.08$ ), $92 \%$ for $x=0.1$ and $c a .80 \%$ for $x \geq 0.2$ The densities of the consolidated pellets were measured using an Archimedes balance ADAM PW184. The consolidated pellets were cut using a MTI SYJ-150 digital diamond saw and carefully polished using a MTI EQ-Unipol-300 grinder/polisher to obtain parallel faces, with less than $0.05 \mathrm{~mm}$ variation in width.

Powder X-ray diffraction data provide evidence of significant preferred orientation in the consolidated materials (Fig. 3). For this reason, physical property measurements were conducted both parallel to the pressing direction, hereafter referred to as cross plane, and perpendicular to the pressing direction, referred to as in plane. The degree of preferred orientation in consolidated materials becomes less marked with increasing values of $x$. This is illustrated by the ratio, $R$, of the intensities of the (001) against the strongest reflection (101) (ESI, $\dagger$ Fig. S1). $R$, which exceeds 30 for $\mathrm{TiS}_{2}$, is reduced to $c a$. 4 for $\mathrm{Co}_{0.5} \mathrm{TiS}_{2}$, where powder pattern simulations indicate ratios of 0.57 and 0.37 respectively in the absence of preferred orientation. Cross-plane thermal diffusivity measurements $(303 \leq T / \mathrm{K} \leq 573)$ were carried out using a Netzsch LFA 447 NanoFlash instrument on polished disks of $c a .2 \mathrm{~mm}$ width and $c a .12 .7 \mathrm{~mm}$ diameter, whilst in-plane measurements $(323 \leq T / \mathrm{K} \leq 573)$ were performed using a Netzsch LFA 457 MicroFlash instrument in $\mathrm{N}_{2}$ atmosphere on plates of ca. $6 \times 6 \times 2 \mathrm{~mm}^{3}$ cut from pellets $8-10 \mathrm{~mm}$ in height. The thermal conductivity was calculated using the Dulong-Petit heat capacity $\left(C_{\mathrm{p}}=0.668 \mathrm{~J} \mathrm{~K}^{-1} \mathrm{~g}^{-1}\right.$ for $\mathrm{TiS}_{2} ; C_{\mathrm{p}}=0.656 \mathrm{~J} \mathrm{~K}^{-1} \mathrm{~g}^{-1}$ for $\left.\mathrm{Co}_{0.10} \mathrm{TiS}_{2}\right)$. In-plane and cross-plane electrical property measurements were made on ingots of $c a .2 \times 2 \times 8-10 \mathrm{~mm}^{3}$, cut from the large pellets.

The temperature dependence of both the electrical resistivity $(\rho)$ and Seebeck coefficient $(S)$ was measured simultaneously over the temperature range $303 \leq T / \mathrm{K} \leq 573$ using a Linseis LSR-3 instrument with samples contained under a partial pressure of He. A temperature gradient of $30 \mathrm{~K}$ was applied across the ends of the ingot for Seebeck coefficient measurements and a $50 \mathrm{~mA}$ current was passed for resistivity measurements.

Simultaneous TGA and DSC analysis of $\mathrm{Co}_{x} \mathrm{TiS}_{2}(x=0,0.08$, $0.25,0.33)$ was carried out using a TA instruments SDT Q600 thermal analyser. The analysis was conducted on $c a .25 \mathrm{mg}$ of finely ground powder over the temperature range $303 \leq T / \mathrm{K} \leq$ 1073 , heating at a rate of $5 \mathrm{~K} \mathrm{~min}^{-1}$. Measurements were made under flows of both nitrogen and air.

\section{Results and discussion}

All investigated compositions of $\mathrm{Co}_{x} \mathrm{TiS}_{2}$ were confirmed to be single phases by powder X-ray diffraction. Powder X-ray diffraction data for $\mathrm{Co}_{x} \mathrm{TiS}_{2}$ phases with $x \leq 0.15$ are well described by a structural model in which cobalt atoms are completely disordered over octahedral sites in the inter-layer space. Observed, calculated and difference profiles are presented as ESI $\dagger$ (Fig. S2). The introduction of inter-layer cobalt cations causes a significant decrease in the $c$-lattice parameter relative to that of $\mathrm{TiS}_{2}$; changes in the in-plane $(a)$ lattice parameter being less marked (Table 1; ESI, $\dagger$ Fig. S3). This behaviour is consistent with previous reports for cobalt intercalation ${ }^{15}$ that differs from most guest-TiS ${ }_{2}$ systems where increasing intercalation levels led to an increase in the $c$ lattice parameter. Recent examples of such systems include $\mathrm{Cu}_{x} \mathrm{TiS}_{2},{ }^{10} \mathrm{Ag}_{x} \mathrm{TiS}_{2}{ }^{8}$ and misfit phases, $(\mathrm{SnS})_{1.2}\left(\mathrm{TiS}_{2}\right)_{2}{ }^{16}$

Whilst powder X-ray diffraction data provide evidence of the formation of superstructures at higher cobalt contents, their detailed structural characterisation using $\mathrm{Cu}-\mathrm{K}_{\alpha}$ radiation is hampered by the increased background signal arising from X-ray fluorescence associated with cobalt. This is particularly marked at low scattering angles $\left(2 \theta \leq 20^{\circ}\right)$, where the relatively weak long $d$-space reflections characteristic of superstructure formation are expected to occur. Therefore, structural characterization of $\mathrm{Co}_{x} \mathrm{TiS}_{2}$ materials with $x \geq 0.2$, was performed using powder neutron diffraction. Powder neutron diffraction data from the

Table 1 Refined structural parameters from Rietveld analysis of powder X-ray diffraction data for $\mathrm{Co}_{x} \mathrm{TiS} \mathrm{S}_{2}$ with $x \leq 0.15$. (space group $P \overline{3} m 1^{a}$ )

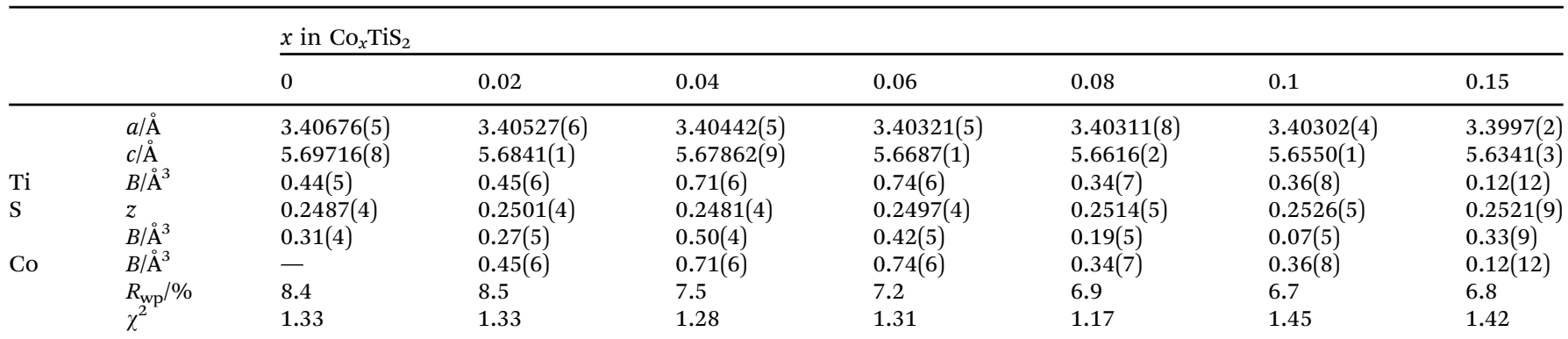

${ }^{a}$ Ti on 1(a), (0, 0, 0); S on 2(d), (1/3, 2/3, z); Co on 1(b) $(0,0,1 / 2)$. SOF(Co) constrained at nominal composition. 
$2 \theta=156^{\circ}, 90^{\circ}, 63^{\circ}$ and $35^{\circ}$ banks of the GEM diffractometer were summed, normalised and used in a multibank Rietveld refinement.

Powder neutron diffraction data for $\mathrm{Co}_{0.25} \mathrm{TiS}_{2}$ can be indexed on a monoclinic unit cell with lattice parameters related to those of the primitive hexagonal sub-cell by $a \approx 2 \sqrt{ } 3 a_{\mathrm{p}}, b \approx 2 a_{\mathrm{p}}, c \approx 2 c_{\mathrm{p}}$. The data are well described (Fig. 4) by a model based on the monoclinic $\mathrm{M}_{5} \mathrm{~S}_{8}(x=1 / 4)$ structure, for which the two-dimensional cation-vacancy ordering scheme is shown schematically in Fig. 2. The powder neutron diffraction data for $\mathrm{Co}_{0.5} \mathrm{TiS}_{2}$ are also indexable on a monoclinic unit cell, but with lattice parameters related to the underlying hexagonal sub-cell through $a \approx \sqrt{ } 3 a_{\mathrm{p}}, b \approx a_{\mathrm{p}}$, $c \approx 2 c_{\mathrm{p}}$. Data (Fig. 4) are well fitted by a structural model based on the $\mathrm{M}_{3} \mathrm{~S}_{4}(x=1 / 2)$ superstructure of Fig. 2, in which $50 \%$ occupancy of interlayer sites results in chains of Co-centered octahedra within the van der Waals' gap of TiS $_{2}$. Refined atomic parameters for the two monoclinic ordered-defect structures are presented in Table 2 .

At $x=0.33$ and $0.40, \mathrm{a} \mathrm{M}_{2} \mathrm{~S}_{3}$-type structure (space group $P \overline{3} 1 c$ ) is adopted with a unit cell related to that of the primitive hexagonal cell by $a \approx \sqrt{ } 3 a_{\mathrm{p}}, b \approx \sqrt{ } 3 a_{\mathrm{p}}, c \approx 2 c_{\mathrm{p}}$. Refined atomic parameters are presented in Table 3. For $x=0.33$, refinement of the site occupancy factor of $\operatorname{Co}(1)$ from its ideal value of unity, was required to produce a satisfactory fit. Allowing for the possibility of cobalt being located at either of the other two octahedral positions, $\operatorname{Co}(2)$ and $\operatorname{Co}(3)$, led to a non-zero site occupancy factor for $\operatorname{Co}(2)(0,0,0.25)$ only. The structure of $\mathrm{Co}_{0.33} \mathrm{TiS}_{2}$ therefore closely approaches the ideal $\mathrm{M}_{2} \mathrm{~S}_{3}$-type with more than $85 \%$ of cobalt at the expected site and a refined stoichiometry of $\mathrm{Co}_{0.31(2)} \mathrm{TiS}_{2}$. The structure of $\mathrm{Co}_{0.4} \mathrm{TiS}_{2}$ is similar, with the additional cobalt cations being distributed over the two octahedral positions, $\operatorname{Co}(2)$ and $\operatorname{Co}(3)$, both normally vacant in $\mathrm{M}_{2} \mathrm{~S}_{3}$. Removing the stoichiometry constraint on site occupancy factors during refinement does not lead to significant deviations from their constrained values, nor does it produce any apparent improvement in the quality of refinement. Final observed, calculated and difference profiles for neutron diffraction data of $\mathrm{Co}_{0.33} \mathrm{TiS}_{2}$ and $\mathrm{Co}_{0.40} \mathrm{TiS}_{2}$ are presented in Fig. 4 .

By contrast, at the extremes of composition investigated using neutron diffraction $(x=0.2,0.67)$, the data (Fig. 5) provide no evidence for ordering of cobalt ions over octahedral sites (Table 4). The data for $\mathrm{Co}_{0.2} \mathrm{TiS}_{2}$ are consistent with a structure (space group $P \overline{3} m 1$ ), analogous to that determined by X-ray methods for $\mathrm{Co}_{x} \mathrm{TiS}_{2}(0.02 \leq x \leq 0.15)$, in which there is a disordered distribution of cobalt ions over the inter-layer octahedral sites. However, a disordered distribution of cobalt ions over inter-layer octahedral sites does not adequately describe the powder neutron diffraction data for $\mathrm{Co}_{0.67} \mathrm{TiS}_{2}$. In addition to higher than expected values of the weighted residual, there is a large discrepancy between the crystallographically determined composition $\left(\mathrm{Co}_{0.43(1)} \mathrm{TiS}_{2}\right)$ and the nominal stoichiometry. The difference Fourier map $\left(\left|F_{\text {obs }}-F_{\text {calc }}\right|\right)$, (Fig. 6), indicates residual nuclear density at positions corresponding to inter-layer tetrahedral sites. Introduction of cobalt into these positions improves the quality of the refinement markedly, and leads to a refined composition $\mathrm{Co}_{0.624(8)} \mathrm{TiS}_{2}$, closer to the nominal stoichiometry,
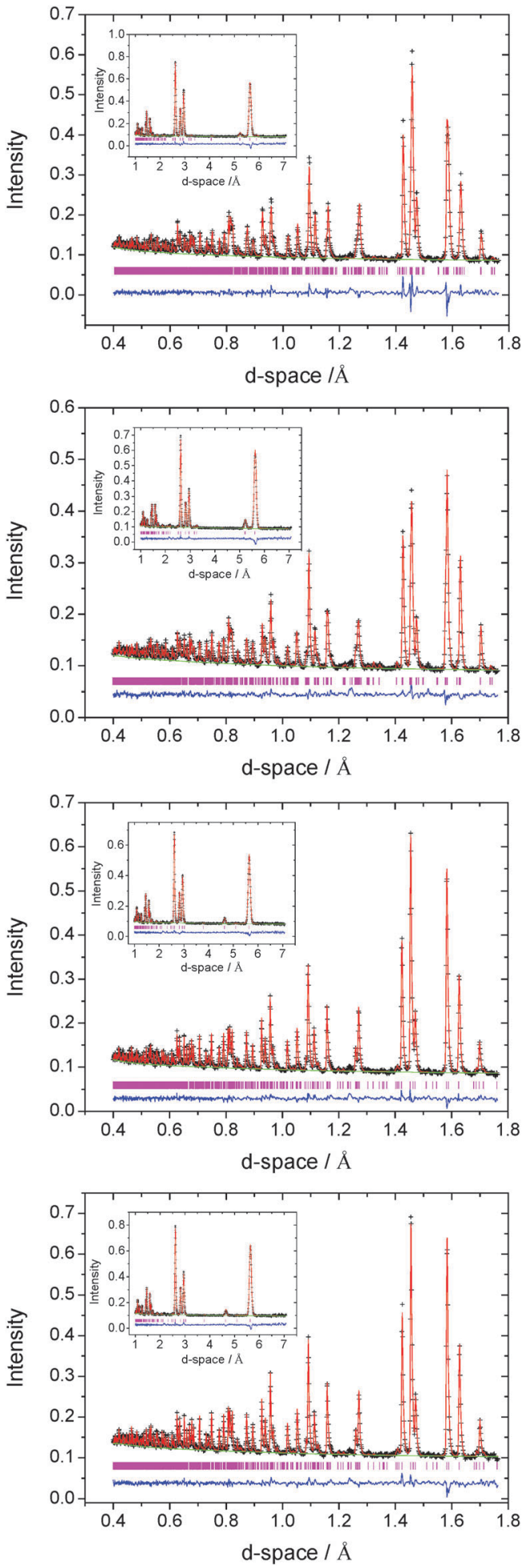

Fig. 4 Observed (crosses), calculated (full line) and difference (lower full line) profiles for $\mathrm{Co}_{x} \mathrm{TiS}_{2}$ with $x=0.25 ; 0.5 ; 0.33 ; 0.4$ from top to bottom, from Rietveld refinement against powder neutron diffraction data from the $2 \theta=156^{\circ}$ detector bank. Data at longer $d$-space $\left(2 \theta=35^{\circ}\right)$ are shown as an inset and reflection positions are marked.

albeit with a slight remaining Co deficiency. To the best of our knowledge this represents a unique example of an $\mathrm{A}_{x} \mathrm{MS}_{2}$ phase in which both octahedral and tetrahedral inter-layer sites are 
Table 2 Refined structural parameters of the ordered defect structures of $\mathrm{CO}_{0.25} \mathrm{TiS}_{2}$ (space group $\left.\mathrm{F} 2 / \mathrm{m}\right)^{a}$ and $\mathrm{CO}_{0.5} \mathrm{TiS}_{2}$ (space group $\left.12 / \mathrm{m}\right)^{b}$

\begin{tabular}{llll}
\hline & & $x$ in $\mathrm{Co}_{x} \mathrm{TiS}_{2}$ & \\
\cline { 2 - 3 } & & 0.25 & 0.50 \\
\hline & $a / \AA$ & $11.7708(4)$ & $5.8943(2)$ \\
& $b / \AA$ & $6.8038(2)$ & $3.40429(9)$ \\
& $c / \AA$ & $11.2444(2)$ & $11.2350(2)$ \\
& $\beta /$ & $90.31(2)$ & $90.278(2)$ \\
$\operatorname{Ti}(1)$ & $x$ & $0.5004(8)$ & $0.0100(5)$ \\
& $z$ & $0.2445(4)$ & $0.2555(2)$ \\
$\operatorname{Ti}(2)$ & $B / \AA^{3}$ & $0.47(2)$ & $0.37(2)$ \\
& $y$ & $0.251(2)$ & - \\
$\mathrm{S}(1)$ & $B / \AA^{3}$ & $0.47(2)$ & $0.3301(5)$ \\
& $x$ & $0.1674(8)$ & $0.3762(2)$ \\
$\mathrm{S}(2)$ & $z$ & $0.1248(5)$ & $0.22(2)$ \\
& $B / \AA^{3}$ & $0.34(2)$ & $0.3344(5)$ \\
$\mathrm{S}(3)$ & $x$ & $0.1640(7)$ & $0.8822(2)$ \\
& $z$ & $0.1157(4)$ & $0.22(2)$ \\
& $B / \AA^{3}$ & $0.34(2)$ & - \\
& $x$ & $0.4173(4)$ & - \\
$\mathrm{Co}$ & $y$ & $0.2491(9)$ & - \\
& $z$ & $0.1245(3)$ & $0.74(3)$ \\
& $B / \AA^{3}$ & $0.34(2)$ & 2.6 \\
& $B / \AA^{3}$ & $1.11(7)$ & 2.05 \\
& $R_{\mathrm{wp}} / \%$ & 3.1 &
\end{tabular}

${ }^{a} \mathrm{Co}(1)$ on $4(\mathrm{a}),(0,0,0)$; $\operatorname{Ti}(1), \mathrm{S}(1), \mathrm{S}(2)$ on $8(\mathrm{i}),(x, 0, z)$; $\operatorname{Ti}(2)$ on $(1 / 4, y$, $1 / 4) ; \mathrm{S}(3)$ on $16(\mathrm{j}),(x, y, z) .{ }^{b} \mathrm{Ti}(1), \mathrm{S}(1), \mathrm{S}(2)$ on $4(\mathrm{i}),(x, 0, z)$; Co(1) on $2(\mathrm{a})$, $(0,0,0)$

Table 3 Refined parameters from Rietveld analysis of powder neutron diffraction data for $\mathrm{Co}_{x} \mathrm{TiS}_{2}, x=0.33,0.40$ (space group $\left.P \overline{3} 1 c\right)^{a}$

\begin{tabular}{llll}
\hline & & $x$ in $\mathrm{Co}_{x} \mathrm{TiS}_{2}$ & \\
\cline { 3 - 3 } & & 0.33 & 0.40 \\
\hline & $a / \AA$ & $5.88111(4)$ & $5.88235(4)$ \\
$\mathrm{Ti}(1)$ & $c / \AA$ & $11.2573(2)$ & $11.2563(2)$ \\
$\mathrm{Ti}(2)$ & $B / \AA^{3}$ & $0.40(2)$ & $0.41(2)$ \\
& $z$ & $-0.0047(2)$ & $-0.0048(3)$ \\
$\mathrm{S}$ & $B / \AA^{3}$ & $0.40(2)$ & $0.41(2)$ \\
& $x$ & $0.333(1)$ & $0.333(1)$ \\
& $y$ & $-0.0006(4)$ & $0.0001(4)$ \\
& $z$ & $0.37192(6)$ & $0.37142(6)$ \\
$\mathrm{Co}(1)$ & $B / \AA^{3}$ & $0.33(2)$ & $0.29(2)$ \\
& $\mathrm{SOF}$ & $0.852(9)$ & 1.0 \\
$\mathrm{Co}(2)$ & $B / \AA^{3}$ & $0.41(5)$ & $0.82(4)$ \\
& $\mathrm{SOF}$ & $0.09(1)$ & $0.151(9)$ \\
$\operatorname{Co}(3)$ & $B / \AA^{3}$ & $0.41(5)$ & $0.82(4)$ \\
& $\mathrm{SOF}$ & - & $0.049(9)$ \\
& $B / \AA^{3}$ & - & $0.82(4)$ \\
& $R_{\mathrm{wp}} / \%$ & 2.7 & 2.7 \\
& $\chi^{2}$ & 1.56 & 1.76
\end{tabular}

${ }^{a} \operatorname{Ti}(1)$ on $2(\mathrm{~d}),(0,0,0)$; $\mathrm{Ti}(2)$ on $4(\mathrm{f}),(1 / 3,2 / 3, z)$; S on $12(\mathrm{i}),(x, y, z)$; $\mathrm{Co}(1)$ on 2 (c), (1/3, 2/3, 0.25); $\mathrm{Co}(2)$ on $2(\mathrm{a}),(0,0,0.25)$; $\mathrm{Co}(3)$ on $2(\mathrm{~b})$, $(2 / 3,1 / 3,0.25)$.

simultaneously occupied. The evolution of the structure of $\mathrm{Co}_{x} \mathrm{TiS}_{2}$ phases with composition, determined from the combination of X-ray and neutron diffraction, is shown schematically in Fig. 7.

Kawasaki et $a l .{ }^{17}$ have recently re-investigated the structural effect of intercalation in $\mathrm{Co}_{x} \mathrm{TiS}_{2}$ using single crystal X-ray diffraction. They described the structure of materials with compositions corresponding to $x=0.26,0.43$ and 0.57 in the trigonal space groups $P \overline{3} m 1(x=0.26,0.57)$ and $P \overline{3} 1 c(x=0.43)$ with unit cells
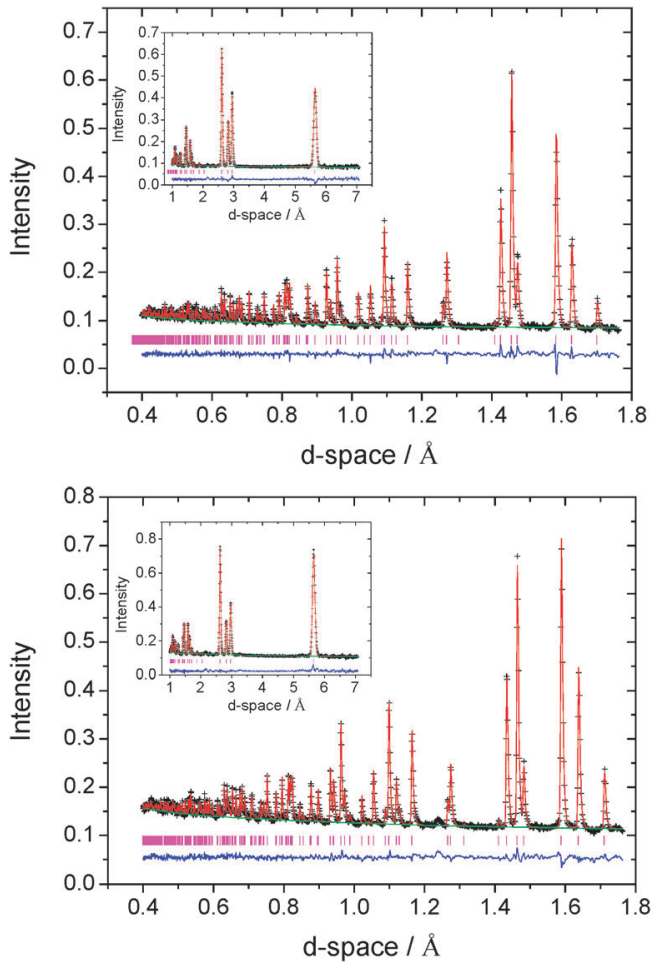

Fig. 5 Observed (crosses), calculated (full line) and difference (lower full line) profiles for $\mathrm{CO}_{0.2} \mathrm{TiS}_{2}$ (top) and $\mathrm{CO}_{0.67} \mathrm{TiS}_{2}$ (bottom) from Rietveld refinement against powder neutron diffraction data from the $2 \theta=156^{\circ}$ detector bank. Data at longer $d$-space $\left(2 \theta=35^{\circ}\right)$ are shown as an inset and reflection positions are marked.

Table 4 Refined parameters from Rietveld analysis of powder neutron diffraction data for $\mathrm{Co}_{x} \mathrm{TiS}_{2}, x=0.2,0.67$ (space group $\left.P \overline{3} \mathrm{~m} 1\right)^{a}$

\begin{tabular}{llll}
\hline & & $x$ in $\mathrm{Co}_{x} \mathrm{TiS}_{2}$ & \\
\cline { 3 - 4 } & & 0.2 & 0.67 \\
\hline \multirow{3}{*}{$\mathrm{Ti}$} & $a / \AA$ & $3.40037(3)$ & $3.42065(3)$ \\
$\mathrm{S}$ & $c / \AA$ & $5.63223(7)$ & $5.64181(8)$ \\
& $B / \AA^{3}$ & $0.56(2)$ & $0.73(2)$ \\
$\mathrm{Co}(1)$ & $z$ & $0.2539(2)$ & $0.2591(2)$ \\
$\mathrm{Co}(2)$ & $B / \AA^{3}$ & $0.43(2)$ & $0.53(2)$ \\
& $\mathrm{SOF}$ & $0.2(-)$ & $0.484(4)$ \\
& $B / \AA^{3}$ & $0.81(8)$ & $0.69(5)$ \\
& $z$ & - & $0.354(3)$ \\
& $\mathrm{SOF}$ & - & $0.070(2)$ \\
& $B / \AA^{3}$ & - & $0.69(5)$ \\
& $R_{\mathrm{wp}} / \%$ & 2.9 & 2.7 \\
& $\chi^{2}$ & 1.70 & 1.95
\end{tabular}

${ }^{a}$ Ti on $1(\mathrm{a}),(0,0,0)$; S on $2(\mathrm{~d}),(1 / 3,2 / 3, z)$; Co(1) on $1(\mathrm{~b}),(0,0,1 / 2)$; $\operatorname{Co}(2)$ on $2(\mathrm{~d}),(2 / 3,1 / 3, z)$.

given by $2 a_{\mathrm{p}} \times 2 a_{\mathrm{p}} \times 2 c_{\mathrm{p}}$ and $\sqrt{ } 3 a_{\mathrm{p}} \times \sqrt{ } 3 a_{\mathrm{p}} \times 2 c_{\mathrm{p}}$ respectively. However, in these models, intercalated cations show a high degree of disorder and those described in $P \overline{3} m 1$ involve an alternation of near fully ordered layers with almost completely disordered layers that is unexpected on energetic grounds. ${ }^{18}$ This contrasts with the conclusions drawn from powder neutron diffraction which shows an evolution of vacancy ordering in every other layer with increasing cobalt content that encompasses 

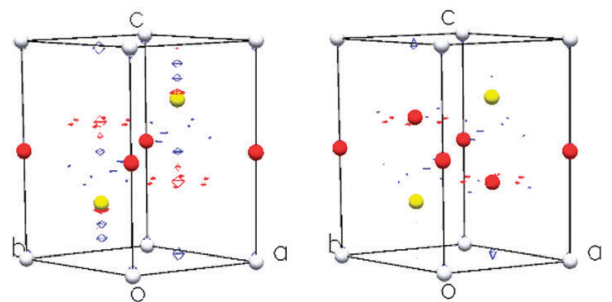

Fig. 6 Difference Fourier maps of $\mathrm{CO}_{0.67} \mathrm{TiS}_{2}$ without (left) and with (right) cobalt in tetrahedral positions. Red and blue contours correspond to positive and negative regions with identical amplitudes.

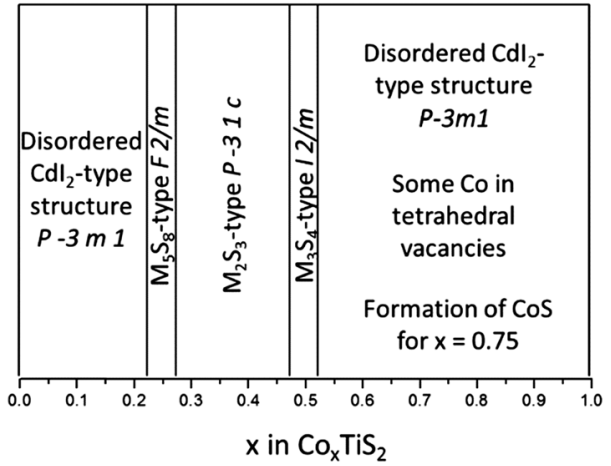

Fig. 7 Structural arrangements of $\mathrm{Co}_{x} \mathrm{TiS}_{2}$ as a function of the cobalt intercalation level, $x$, established from XRD and neutron diffraction analysis.

monoclinic $\mathrm{M}_{5} \mathrm{~S}_{8}$, trigonal $\mathrm{M}_{2} \mathrm{~S}_{3}$ and monoclinic $\mathrm{M}_{3} \mathrm{~S}_{4}$ structures, some of which are stabilised over a range of compositions, in addition to disordered structures observed at the compositional extremes. Support for the conclusions drawn here is provided by previous studies ${ }^{19-21}$ which suggest monoclinic distortions in phases with $x=0.25$ and 0.5 for $\mathrm{Co}_{x} \mathrm{TiS}_{2}$.
Magnetic susceptibility data (Fig. 8) provide further insights into the electronic states of these phases. The susceptibility data for $\mathrm{Co}_{0.20} \mathrm{TiS}_{2}$ clearly reveal a ferromagnetic transition at $T_{\mathrm{C}}=130 \mathrm{~K}$, although no magnetic scattering was detectable in powder neutron diffraction data collected at $2 \mathrm{~K}$. This may be a consequence of the small ordered moment arising from the $20 \%$ occupancy of the $\operatorname{Co}(1)$ site. Inoue et $a l^{22}$ have observed a similar transition in AC susceptibility data for single crystals of $\mathrm{Co}_{x} \mathrm{TiS}_{2}(0.075 \leq x \leq 0.33)$. In the present work no magnetic ordering transitions were observed for $x=0.25$ and $x=0.33$, which may be attributable to differences in the homogeneity of the single crystal and powder samples. However, it is possible that these previous studies and the present $\mathrm{Co}_{0.2} \mathrm{TiS}_{2}$ sample contain trace amounts of $\mathrm{CoS}_{2}$, which is a ferromagnet with a Curie temperature reported between $110 \mathrm{~K}$ and $130 \mathrm{~K}^{23-25}$ The magnetic susceptibility decreases with increasing cobalt content up to $x=0.66$, consistent with the work of Danot et al. ${ }^{21}$ Data in the paramagnetic regions are well described by a modified Curie-Weiss law $\left(\chi=\chi_{0}+C / T-\theta\right)$ incorporating a temperature independent term. At low cobalt contents, the effective magnetic moments (Table 5) are in reasonable agreement with the spinonly values for low-spin octahedral $\mathrm{Co}^{2+}\left(\mu_{\mathrm{eff}}=1.73\right)$. However, as the cobalt content increases above $x=0.33$, the moment deviates significantly from spin-only behaviour. This may be attributed to an increasing degree of electron delocalization as the concentration of cobalt in the vacancy layer increases. This observation supports the conclusion previously drawn by Inoue et al. ${ }^{1,22}$ on the formation of a $\left(\mathrm{t}_{2 \mathrm{~g}}\right)^{6}\left(\mathrm{e}_{\mathrm{g}}\right)^{1}$ low-spin band near the Ti 3d band. The negative Weiss constant indicates that in all phases for $x \leq 0.66$, the dominant magnetic exchange interactions are antiferromagnetic in origin with a weak ferromagnetic component observed for $\mathrm{Co}_{0.25} \mathrm{TiS}_{2}$ and $\mathrm{Co}_{0.66} \mathrm{TiS}_{2}$. Antiferromagnetic correlations may also serve to reduce the effective magnetic moment as evidenced by the decrease in the quantity
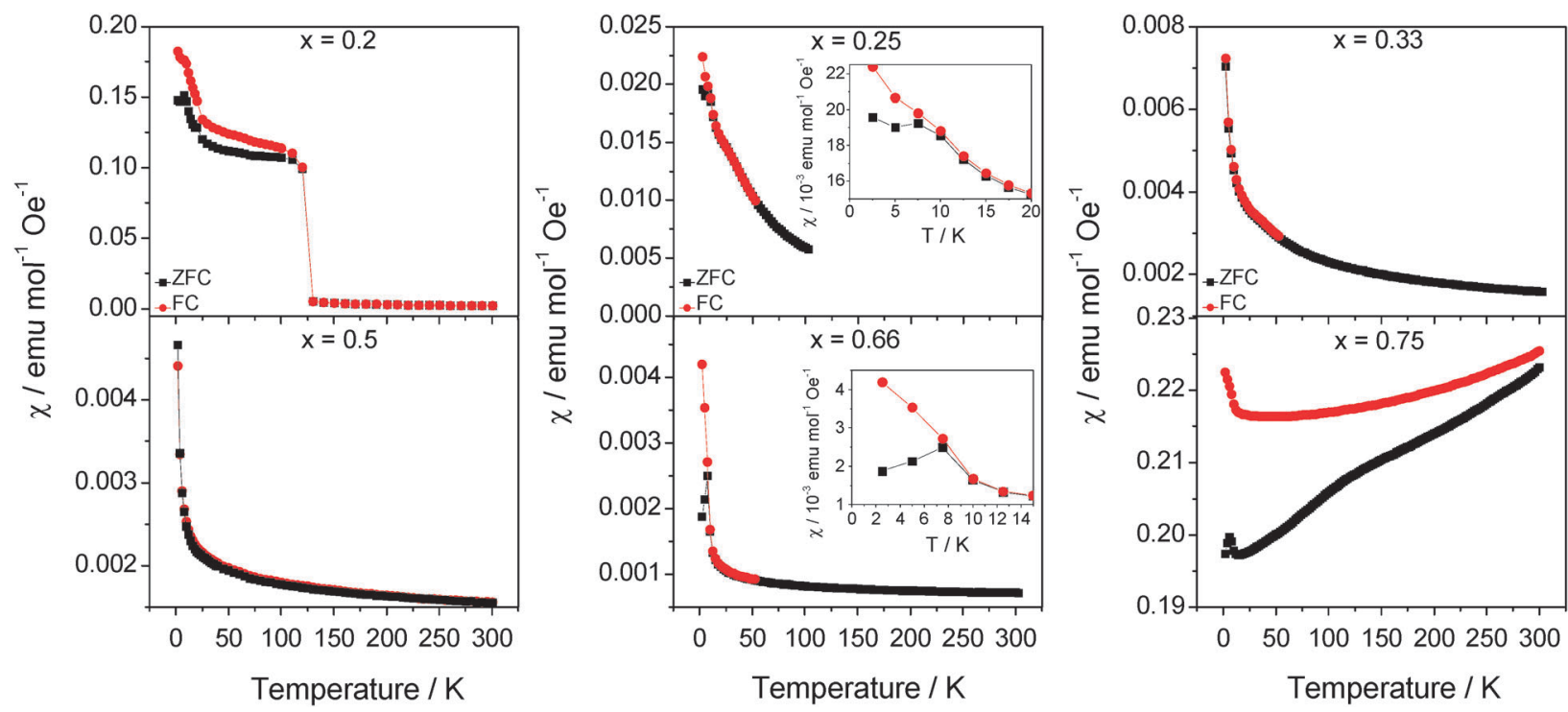

Fig. 8 Zero-field cooled (black squares) and field-cooled (red circles) molar magnetic susceptibility of $\mathrm{Co}_{x} \mathrm{TiS}_{2}(x=0.2 ; 0.25 ; 0.33 ; 0.5 ; 0.66$ and 0.75$)$. Insets show details in the region of anomalies in $\chi_{\text {mol }}(T)$. 
Table 5 Derived magnetic parameters obtained from the fit of a modified Curie-Weiss law to magnetic susceptibility data over the temperature range specified

\begin{tabular}{lllll}
\hline$x$ & Temp. range $(\mathrm{K})$ & $\chi_{0}\left(\mathrm{emu} \mathrm{mol}^{-1}\right)$ & $\theta(\mathrm{K})$ & $\mu_{\text {eff }}$ per Co \\
\hline 0.2 & $150-300$ & - & $-26(3)$ & $2.36(7)$ \\
0.25 & $40-100$ & $3.91(8) \times 10^{-4}$ & $-13.5(5)$ & $2.24(3)$ \\
0.33 & $40-300$ & $1.100(7) \times 10^{-3}$ & $-36(1)$ & $1.15(4)$ \\
0.5 & $55-215$ & $1.430(6) \times 10^{-3}$ & $-45(2)$ & $0.63(1)$ \\
0.67 & $25-220$ & $6.70(2) \times 10^{-4}$ & $-17(2)$ & $0.37(3)$ \\
\hline
\end{tabular}

$(8 \chi T / n)^{1 / 2}$ with decreasing temperature (ESI, $\dagger$ Fig. S4). At a composition $\mathrm{Co}_{0.75} \mathrm{TiS}_{2}$ very different behaviour is observed in the magnetic susceptibility data. The data appear to indicate a magnetic ordering transition that occurs above $300 \mathrm{~K}$ - this may be estimated as $350-400 \mathrm{~K}$ (ESI, $\dagger$ Fig. S5). This may be associated with the presence of trace amounts of $\operatorname{CoS}\left(T_{\mathrm{N}}=358 \mathrm{~K}\right)^{24}$ suggesting that the limit of the single-phase region of $\mathrm{Co}_{x} \mathrm{TiS}_{2}$ lies in the range $0.66 \leq x \leq 0.75$.

TGA/DSC analysis of $\mathrm{Co}_{x} \mathrm{TiS}_{2}(x=0 ; 0.08 ; 0.25$ and 0.33$)$, performed under both inert $\left(\mathrm{N}_{2}\right)$ and oxidising (air) atmospheres, are provided as ESI $\dagger$ (Fig. S6). All four samples produce an exothermic peak when heated in air, corresponding to rapid oxidation. Intercalation of cobalt shifts the exothermic peak to higher temperature; from $615 \mathrm{~K}$ for $\mathrm{TiS}_{2}$ to $713 \mathrm{~K}$ for $\mathrm{Co}_{0.33} \mathrm{TiS}_{2}$. Powder X-ray diffraction (ESI, $\dagger$ Fig. S7 and S8) identifies the oxidation products as being anatase $\mathrm{TiO}_{2}$ for $x=0$; anatase with evidence for the rutile form at $x=0.08$; and a mixture of rutile and $\mathrm{CoTiO}_{3}$ for higher cobalt contents. In an inert atmosphere, no exotherms arising from oxidation are observed, however a slow oxidation due to the presence of trace amounts of oxygen occurs over the $160 \mathrm{~min}$ of the thermal analysis run, leading to formation of rutile and anatase $\mathrm{TiO}_{2}$ in addition to the $\mathrm{Co}_{x} \mathrm{TiS}_{2}$ phases. For $\mathrm{TiS}_{2}$ and $\mathrm{Co}_{0.08} \mathrm{TiS}_{2}$, weight loss associated with sulphur volatilisation occurs with an onset in the range $473 \leq$ $T / \mathrm{K} \leq 573$, suggesting that a protective coating would be required for applications at temperatures above $573 \mathrm{~K}$. To avoid degradation of the samples through sulphur volatilization, all transport measurements were therefore performed at temperatures below $573 \mathrm{~K}$.

The Seebeck coefficient (Fig. 9) determined here for polycrystalline $\mathrm{TiS}_{2}$ at $300 \mathrm{~K}$ is in good agreement with the measurements by Imai et $a .^{7}$ on single crystals: The value of $S=-232 \mu \mathrm{V} \mathrm{K}^{-1}$ in the present study for the in-plane direction compares favourably that of $S=-251 \mu \mathrm{V} \mathrm{K}^{-1}$ at $300 \mathrm{~K}$ within the $a b$ plane in single crystals. Similarly, $\rho=0.034 \mathrm{~m} \Omega \mathrm{m}$ in the in-plane direction for the hot pressed sample is of the same order of magnitude to that determined in the $a b$ plane for the single crystals $(\rho=0.017 \mathrm{~m} \Omega \mathrm{m})$. Notably, the cross-plane resistivity is reduced by two orders of magnitude from that along the crystallographic $c$ direction in the single crystal, indicating the less than perfect alignment of plate-like crystallites in the hot-pressed polycrystalline sample. The electrical properties of $\mathrm{TiS}_{2}$ have been the subject of some debate as discrepancies in physical properties determined by different groups led to uncertainty over whether $1 \mathrm{~T}-\mathrm{TiS}_{2}$ is a semi-metal $^{26,27}$ or a semiconductor, with different values of direct or indirect band gap having been reported. However, it is

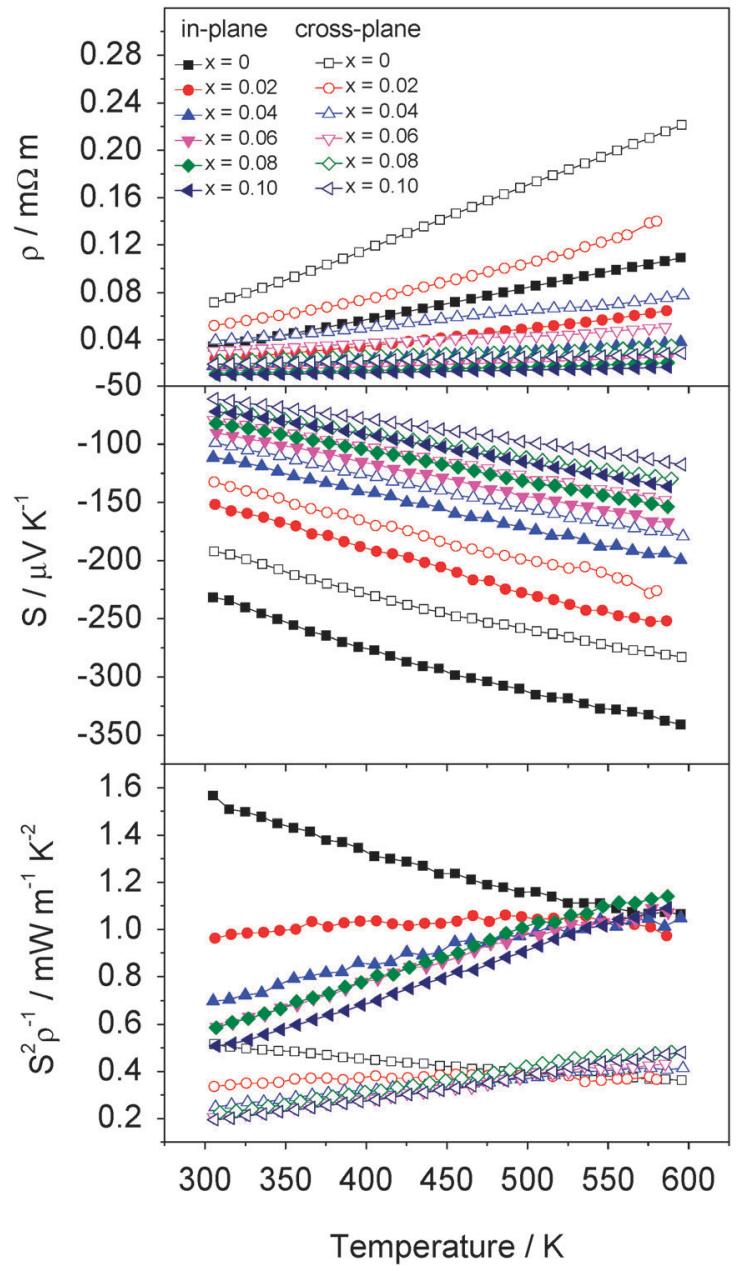

Fig. 9 Temperature dependence of the electrical resistivity $(\rho)$, Seebeck coefficient $(S)$ and power factor $\left(S^{2} \rho^{-1}\right)$ of $\mathrm{Co}_{x} \mathrm{TiS}_{2}(0 \leq x \leq 0.10)$. Full symbols represent measurements made along the in-plane direction while the empty symbols correspond to cross-plane measurements.

now understood that the origin of the variations in physical properties lies in sulphur volatilisation at high temperatures. This results in titanium self-intercalation into the van der Waals' gap. The properties of the resulting phases formulated $\mathrm{Ti}_{1+\gamma} \mathrm{S}_{2}$, are sensitively dependent on the level of non-stoichiometry. ${ }^{9,28,29}$ In an effort to minimise the effects of sulphur volatilization and the resulting self-intercalation of titanium, controlled cooling was adopted in the present work and identical conditions were adopted for synthesis and consolidation of all samples The success of this approach is reflected in the Seebeck coefficient, the magnitude of which provides a sensitive probe of the degree of non-stoichiometry in the parent phase. ${ }^{9}$ Comparison between in-plane measurements reported here and those for $\mathrm{Ti}_{1+\gamma} \mathrm{S}_{2}$ that we have previously reported provides an estimate of the upper limit of non-stoichiometry of $1.5 \%$, corresponding to a composition $\mathrm{Ti}_{1.015} \mathrm{~S}_{2}$. This is also consistent with a $c$ lattice parameter of $<5.70 \AA$ and a unit cell volume, $V<57.3 \AA^{3}$, both of which suggest the deviation from stoichiometry lies in the range $1-1.5 \%$.

Electrical resistivity and Seebeck coefficient measurements for $\mathrm{Co}_{x} \mathrm{TiS}_{2}$ over the range of composition $0 \leq x \leq 0.4$ were 
initially carried out in plane (Fig. S9, ESI $\dagger$ ). These measurements reveal that both the electrical resistivity and the absolute value of the Seebeck coefficient decrease markedly with increasing $x$. This can be attributed to the donation of electrons from the cobalt cation to the empty $\mathrm{t}_{2 \mathrm{~g}}$-derived conduction band of $\mathrm{TiS}_{2}$, thereby increasing the charge carrier density at $E_{\mathrm{F}}$. This results in thermoelectric power factors below $0.3 \mathrm{~mW} \mathrm{~m}^{-1} \mathrm{~K}^{-2}$ for materials with compositions corresponding to $x \geq 0.2$. By contrast, at lower cobalt contents, the power factor reaches values that are comparable to that of polycrystalline $\mathrm{TiS}_{2}$. Similarly, cross-plane thermal conductivity measurements for $\mathrm{Co}_{x} \mathrm{TiS}_{2}$ samples with $x$ $>0.1$, are significantly higher than those for materials with lower Co contents. This may be attributed in part to an increase in charge carrier concentration due to the transfer of electrons from Co to the $\mathrm{TiS}_{2}$ host. However, the electronic contribution to the thermal conductivity $\left(\kappa_{\mathrm{el}}\right),(\mathrm{ESI}, \dagger$ Fig. S10) calculated from the Wiedemann-Franz law decreases again above $x=0.25$ due to the increased electrical resistivity which may be associated with the lower level of densification of materials with higher cobalt levels. The overall thermal conductivity however remains high for these samples despite an increased porosity, (ESI, $\uparrow$ Fig. S11) suggesting that the lattice contribution also increases with cobalt intercalation. For these reasons, the thermoelectric properties of samples with higher Co contents were not investigated in further detail and henceforth we focus on the thermoelectric behaviour of $\mathrm{Co}_{x} \mathrm{TiS}_{2}$ with $x \leq 0.10$.

The electrical resistivity measured in the in-plane and in the cross-plane directions of $\mathrm{Co}_{x} \mathrm{TiS}_{2}(x \leq 0.10)$ (Fig. 9) decreases with decreasing temperature: behaviour reminiscent of a metal. The Seebeck coefficient of $\mathrm{Co}_{x} \mathrm{TiS}_{2}$ materials is negative for all values of $x$ with the absolute value increasing with $T$. This is consistent with the dominant charge carriers being electrons in all materials studied. Despite the apparent metal-like temperature dependence of the resistivity, values of the Seebeck coefficient are generally considerably higher than anticipated for a conventional metal, suggesting that the materials are better described as degenerate semiconductors. Both electrical resistivity and Seebeck coefficient are higher for cross-plane measurements compared to in-plane measurements from the same pellet. This anisotropy is attributed to the 2-dimensional layered structure and the preferential alignment of the crystallites during consolidation.

Whilst increasing cobalt intercalation reduces both the electrical resistivity and Seebeck coefficient, at low cobalt contents the gain from the former is sufficient at higher temperatures to compensate for the impact of the reduction in Seebeck coefficient. This produces a comparable thermoelectric power factor $\left(S^{2} \rho^{-1}\right)$ to that of the pristine phase (Fig. 9) at temperatures above $500 \mathrm{~K}$ for cross-plane and above $575 \mathrm{~K}$ for in-plane measurements. The power factor for $\mathrm{Co}_{x} \mathrm{TiS}_{2}(0 \leq x \leq 0.10)$ reaches ca. $1-1.1 \mathrm{~mW} \mathrm{~m}^{-1} \mathrm{~K}^{-2}$ over the $550 \mathrm{~K}$ to $595 \mathrm{~K}$ range in the in-plane direction, while the cross-plane electrical properties are significantly lowered by the preferred orientation and the anisotropy highlighted by the single-crystal properties with power factors ranging from 0.35 to $0.45 \mathrm{~mW} \mathrm{~m}^{-1} \mathrm{~K}^{-2}$ over the $500 \mathrm{~K}$ to $595 \mathrm{~K}$ range.

In contrast with the behaviour of $\mathrm{Co}_{x} \mathrm{TiS}_{2}$ materials with high cobalt contents discussed above, significant reductions in thermal conductivity may be realised at compositions corresponding to $x \leq 0.08$ (Fig. 10). Thermal conductivity data reveal that these reductions are achieved despite the increased charge carrier contribution $\left(\kappa_{\mathrm{el}}\right)$, as a consequence of electrons donated from cobalt. Subtraction of this charge carrier contribution, estimated from the Wiedemann-Franz law $\left(L=2.44 \times 10^{-8} \mathrm{~W} \Omega \mathrm{K}^{-2}\right)$ reveals that the introduction of low levels of cobalt into the inter-layer space of $\mathrm{TiS}_{2}$ effects marked reductions in the lattice contribution to the thermal conductivity $\left(\kappa_{\mathrm{L}}\right)$. This suggests that a disordered array of cobalt ions in the van der Waals' gap of the metal dichalcogenide structure provides an effective means of scattering heat carrying phonons. The effect is particularly marked along the in-plane direction with $\kappa$ decreasing by $20 \%$ on going from the pristine phase to $\mathrm{Co}_{0.04} \mathrm{TiS}_{2}$.

The compositional dependence of the in-plane and crossplane electrical resistivity, Seebeck coefficient, power factor, lattice and total thermal conductivities and figure of merit for $\mathrm{Co}_{x} \mathrm{TiS}_{2}(x \leq 0.1)$ at selected temperatures is shown in Fig. 11 . For all compositions, the in-plane and cross-plane properties exhibit a similar compositional dependence. A rapid fall in electrical resistivity and the corresponding decrease of $|S|$ with increasing cobalt content results in a change in the temperature dependence of the power factor at $x=0.02$ from a negative to a positive gradient. The lattice contribution to the thermal conductivity also decreases rapidly with cobalt intercalation up to $x=0.08$ with a slightly more pronounced effect in the in-plane than in the cross plane

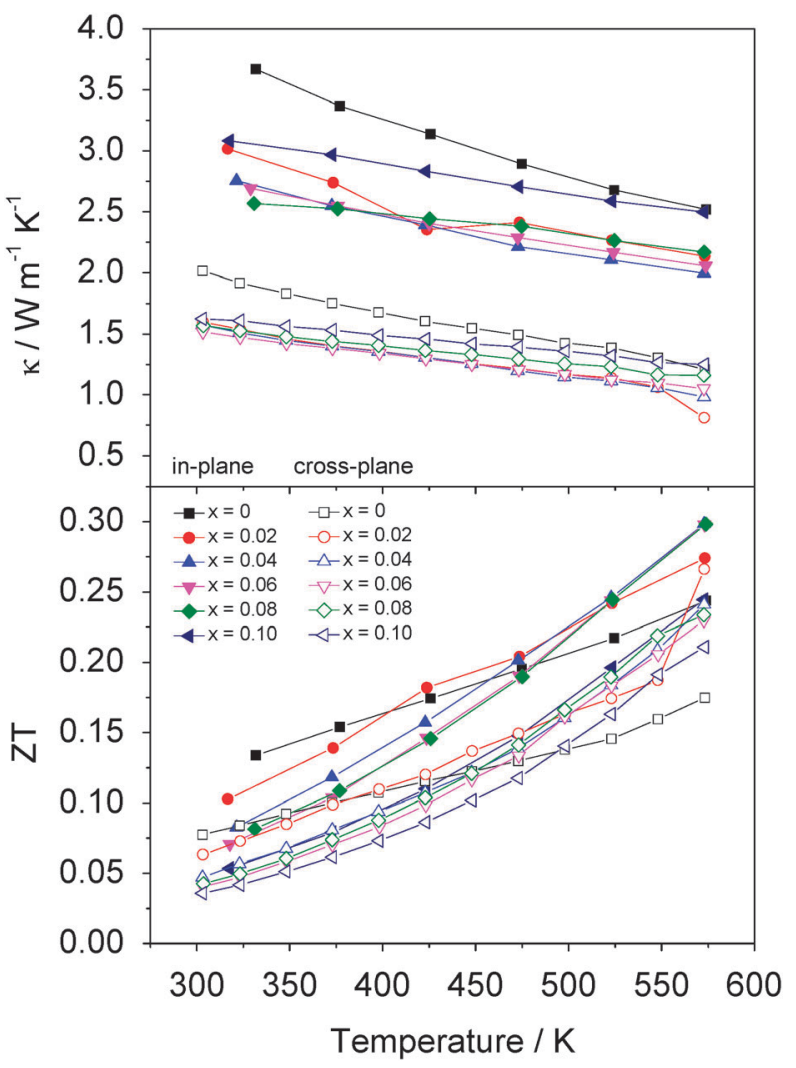

Fig. 10 Temperature dependence of the thermal conductivity (top) and $Z T$ (bottom) of $\mathrm{Co}_{x} \mathrm{TiS}_{2}$. $(0 \leq x \leq 0.10)$. In-plane and cross plane data are indicated by solid and open symbols respectively. 

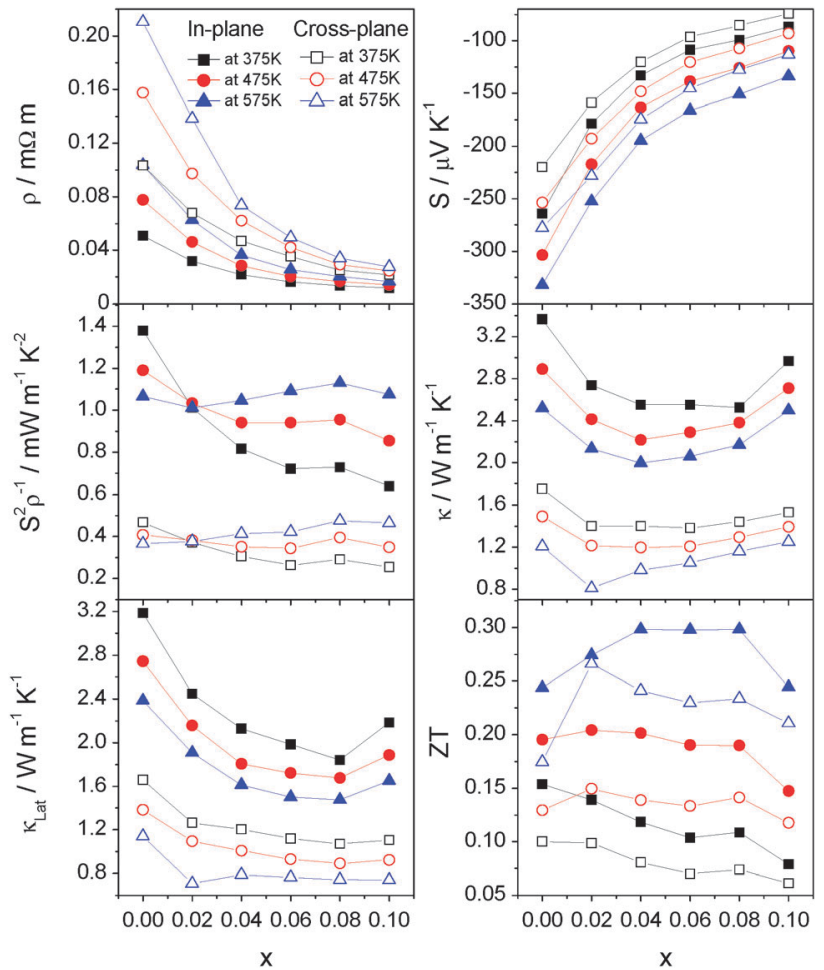

Fig. 11 Compositional dependence of the in-plane (solid symbols) and crossplane (open symbols) thermoelectric performance of $\mathrm{Co}_{x} \mathrm{TiS}_{2}(0 \leq x \leq 0.10)$, including electrical resistivity (top left), Seebeck coefficient (top right), power factor (middle left), thermal conductivity (middle right), electronic contribution to thermal conductivity (bottom left) and ZT (bottom right).

direction. Despite the rapid rise in the electronic contribution, the total thermal conductivity is reduced and a flat minimum is observed over the range $0.02 \leq x \leq 0.06$. The maintenance of a reasonably high power factor on intercalation combined with a reduced thermal conductivity produces an enhancement of $Z T$ over that of $\mathrm{TiS}_{2}$. Whilst all samples with low levels of intercalation $(x \leq 0.08)$ show an enhancement over the temperature range investigated, the best performance is achieved for materials with compositions in the range $0.04 \leq x \leq 0.08$, that exhibit $Z T=0.3$ at $573 \mathrm{~K}$ in the in-plane direction, a $25 \%$ increase over the maximum value of that for $\mathrm{TiS}_{2}$ at the same temperature. The effect of divalent cobalt intercalation is similar to that of monovalent copper $^{10,30}$ and silver ${ }^{8}$ with intercalation simultaneously decreasing the electrical resistivity and thermal conductivity. The maximum $Z T=0.3$ reported here for hot-pressed samples compares favourably with $\mathrm{M}_{x} \mathrm{TiS}_{2}(\mathrm{M}=\mathrm{Cu}, \mathrm{Ag}$, Ti) at $573 \mathrm{~K}$ prepared by SPS.

\section{Conclusions}

Using a combination of powder X-ray and neutron diffraction, we have established a structural phase diagram of $\mathrm{Co}_{x} \mathrm{TiS}_{2}$ $(0 \leq x \leq 0.75)$ which exhibits, with increasing cobalt content an evolution from a disordered phase through three superstructure types, monoclinic $\mathrm{M}_{5} \mathrm{~S}_{8}$, trigonal $\mathrm{M}_{2} \mathrm{~S}_{3}$ and monoclinic $\mathrm{M}_{3} \mathrm{~S}_{4}$, to a second disordered region at the highest cobalt contents in which both octahedral and tetrahedral sites are occupied. The intercalation of cobalt causes marked changes to the electrontransport properties of $\mathrm{TiS}_{2}$. In particular, electron transfer from cobalt to the conduction band of the $\mathrm{TiS}_{2}$ host results in a systematic reduction in the electrical resistivity in well-densified samples. Whilst the lower resistivity is advantageous from a thermoelectric perspective, the accompanying fall in $|S|$ leads to low power factors in phases with higher cobalt contents. Moreover, the thermal conductivity remains high for samples with high cobalt content despite the increased porosity of the samples and the artificially low electronic contribution. Conversely, at low levels of cobalt incorporation, $(x \leq 0.10)$, the gain in electrical conductivity is sufficiently high to offset the reduction in the absolute value of the Seebeck coefficient, leading to a power factor comparable with or higher than that of pristine $\mathrm{TiS}_{2}$ over the $\mathrm{Co}_{x} \mathrm{TiS}_{2}$ series $(0.02 \leq x \leq 0.1)$ at temperatures above $500 \mathrm{~K}$ for cross-plane and above $550 \mathrm{~K}$ for in-plane measurements. The disordered arrangement of cobalt cations over inter-layer octahedral sites also leads to a reduction in lattice thermal conductivity, $\kappa_{\mathrm{L}}$, through increased phonon scattering. The decrease in $\kappa_{\mathrm{L}}$ ameliorates the impact of the increased charge carrier contribution, leading to a decrease in overall thermal conductivity. The changes induced by incorporation of low levels of cobalt increases the figure of merit by $c a .25 \%$ over that of $\mathrm{TiS}_{2}$, with $Z T$ reaching a maximum $Z T=0.3$ for $\mathrm{Co}_{x} \mathrm{TiS}_{2}(0.04 \leq x \leq 0.08)$ at $573 \mathrm{~K}$. Whilst higher $Z T$ values have been reported for n-type sulphides, this level of performance is achieved at considerably higher temperatures with $Z T \geq 0.4$ at $950 \mathrm{~K}$ for $\mathrm{Cu}_{4} \mathrm{Mo}_{6} \mathrm{~S}_{8}{ }^{31}$ and $\mathrm{LaGd}_{1.02} \mathrm{~S}_{3},{ }^{32}$ and $Z T \geq 1$ for doped- $\mathrm{Bi}_{2} \mathrm{~S}_{3}$ at $923 \mathrm{~K} \cdot{ }^{33}$ For the temperature range appropriate to energy recovery from low grade waste heat that is the focus of the work described here, the maximum $Z T$ attained is similar to that of $\mathrm{M}_{x} \mathrm{TiS}_{2}(\mathrm{M}=\mathrm{Cu}, \mathrm{Ag})^{8,10}$ and $\mathrm{TiS}_{2}$-based superlattices ${ }^{3,11}$ prepared by Spark Plasma Sintering and to the hot-pressed Shandite-type phase $\mathrm{Co}_{3} \mathrm{Sn}_{1.6} \mathrm{In}_{0.4} \mathrm{~S}_{2} \cdot{ }^{34}$ The recent report of comparable performance in a p-type synthetic bornitetype sulphide ${ }^{35}$ suggests that with further development, sulphidebased thermoelectrics may offer an attractive alternative to $\mathrm{Bi}_{2} \mathrm{Te}_{3}$ for thermoelectric energy recovery from waste heat at low temperatures using earth-abundant elements.

\section{Acknowledgements}

Financial support from the European Commission (FP7-SME2012-1, Grant Agreement No. 315019) is gratefully acknowledged. The authors wish to thank the University of Reading for financial support for GG and for access to the Chemical Analysis Facility for powder X-ray diffraction and TGA/DSC. The authors would also like to express their gratitude to Dr Gavin Stenning, STFC, ISIS Facility for his help with SQUID measurements.

\section{Notes and references}

1 M. Inoue, H. P. Hughes and A. D. Yoffe, Adv. Phys., 1989, 38, 565-604.

2 G. A. Wiegers and R. J. Haange, Eur. J. Solid State Inorg. Chem., 1991, 28, 1071-1078. 
3 C. Wan, Y. Wang, N. Wang and K. Koumoto, Materials, 2010, 3, 2606-2617.

4 C. Wan, X. Gu, F. Dang, T. Itoh, Y. Wang, H. Sasaki, M. Kondo, K. Koga, K. Yabuki, G. J. Snyder, R. Yang and K. Koumoto, Nat. Mater., 2015, 14, 622-627.

5 D. M. Rowe, in Thermoelectrics Handbook: Macro to Nano, ed. D. M. Rowe, CRC Press, 2006, ch. 1, pp. 1.1-1.13.

6 G. J. Snyder and E. S. Toberer, Nat. Mater., 2008, 7, 105-114.

7 H. Imai, Y. Shimakawa and Y. Kubo, Phys. Rev. B: Condens. Matter Mater. Phys., 2001, 64, 241104.

8 T. Barbier, O. I. Lebedev, V. Roddatis, Y. Breard, A. Maignan and E. Guilmeau, Dalton Trans., 2015, 44, 7887-7895.

9 M. Beaumale, T. Barbier, Y. Breard, G. Guelou, A. V. Powell, P. Vaqueiro and E. Guilmeau, Acta Mater., 2014, 78, 86-92.

10 E. Guilmeau, Y. Breard and A. Maignan, Appl. Phys. Lett., 2011, 99, 052107.

11 E. Guilmeau, A. Maignan, C. Wan and K. Koumoto, Phys. Chem. Chem. Phys., 2015, 17, 24541-24555.

12 O. Arnold, J. C. Bilheux, J. M. Borreguero, A. Buts, S. I. Campbell, L. Chapon, M. Doucet, N. Draper, R. Ferraz Leal, M. A. Gigg, V. E. Lynch, A. Markvardsen, D. J. Mikkelson, R. L. Mikkelson, R. Miller, K. Palmen, P. Parker, G. Passos, T. G. Perring, P. F. Peterson, S. Ren, M. A. Reuter, A. T. Savici, J. W. Taylor, R. J. Taylor, R. Tolchenov, W. Zhou and J. Zikovsky, Nucl. Instrum. Methods Phys. Res., Sect. A, 2014, 764, 156-166.

13 A. C. Larson, R. B. V. Dreele, Los Alamos National Laboratory Report LAUR 86-748, 1994.

14 L. W. Finger, M. Kroeker and B. H. Toby, J. Appl. Crystallogr., 2007, 40, 188-192.

15 M. Inoue and H. Negishi, J. Phys. Chem., 1986, 90, 235-238.

16 C. Wan, Y. Wang, N. Wang, W. Norimatsu, M. Kusunoki and K. Koumoto, J. Electron. Mater., 2011, 40, 1271-1280.

17 T. Kawasaki and K.-I. Ohshima, J. Phys. Soc. Jpn., 2011, 80, 044601.

18 K. Kosuge, Chemistry of Non-Stoichiometric Compounds, Oxford University Press, Oxford, 1993.
19 M. Danot and R. Brec, Acta Crystallogr., Sect. B: Struct. Crystallogr. Cryst. Chem., 1975, 31, 1647-1652.

20 M. Danot and J. Rouxel, C. R. Hebd. Seances Acad. Sci., 1970, 271, 998-1001.

21 M. Danot, J. Rouxel and O. Gorochov, Mater. Res. Bull., 1974, 9, 1383-1392.

22 M. Inoue, M. Matsumoto, H. Negishi and H. Sakai, J. Magn. Magn. Mater., 1985, 53, 131-138.

23 L. Neel and R. Benoit, C. R. Hebd. Seances Acad. Sci., 1953, 237, 444-447.

24 J. B. Goodenough, Magnetism and the Chemical Bond, Wiley, New York, 1963.

25 S. Miyahara and T. Teranishi, J. Appl. Phys., 1968, 39, 896-897.

26 A. H. Reshak and S. Auluck, Phys. Rev. B: Condens. Matter Mater. Phys., 2003, 68, 245113.

27 C. M. Fang, R. A. de Groot and C. Haas, Phys. Rev. B: Condens. Matter Mater. Phys., 1997, 56, 4455-4463.

28 P. C. Klipstein, A. G. Bagnall, W. Y. Liang, E. A. Marseglia and R. H. Friend, J. Phys. C: Solid State Phys., 1981, 14, 4067-4081.

29 M. Beaumale, T. Barbier, Y. Breard, S. Hebert, Y. Kinemuchi and E. Guilmeau, J. Appl. Phys., 2014, 115, 043704.

30 A. Maignan, E. Guilmeau, F. Gascoin, Y. Breard and V. Hardy, Sci. Technol. Adv. Mater., 2012, 13, 053003.

31 M. Ohta, H. Obara and A. Yamamoto, Mater. Trans., 2009, 50, 2129-2133.

32 M. Ohta, S. Hirai and T. Kuzuya, J. Electron. Mater., 2011, 40, 537-542.

33 L.-D. Zhao, S.-H. Lo, J. He, H. Li, K. Biswas, J. Androulakis, C.-I. Wu, T. P. Hogan, D.-Y. Chung, V. P. Dravid and M. G. Kanatzidis, J. Am. Chem. Soc., 2015, 133, 20476-20487.

34 J. Corps, P. Vaqueiro, A. Aziz, R. Grau-Crespo, W. Kockelmann, J.-C. Jumas and A. V. Powell, Chem. Mater., 2015, 27, 3946-3956.

35 G. Guélou, A. V. Powell and P. Vaqueiro, J. Mater. Chem. C, 2015, 3, 10624-10629. 